\title{
Efficacy of amantadine on behavioural problems due to acquired brain injury
}

Citation for published version (APA):

Ter Mors, B. J., Backx, A. P. M., Spauwen, P., Ponds, R. W. H. M., Van Harten, P. N., \& Van Heugten, C. M. (2019). Efficacy of amantadine on behavioural problems due to acquired brain injury: A systematic review. Brain Injury, 33(9), 1137-1150. https://doi.org/10.1080/02699052.2019.1631482

Document status and date:

Published: 29/07/2019

DOI:

10.1080/02699052.2019.1631482

Document Version:

Publisher's PDF, also known as Version of record

Document license:

Taverne

Please check the document version of this publication:

- A submitted manuscript is the version of the article upon submission and before peer-review. There can be important differences between the submitted version and the official published version of record.

People interested in the research are advised to contact the author for the final version of the publication, or visit the DOI to the publisher's website.

- The final author version and the galley proof are versions of the publication after peer review.

- The final published version features the final layout of the paper including the volume, issue and page numbers.

Link to publication

\footnotetext{
General rights rights.

- You may freely distribute the URL identifying the publication in the public portal. please follow below link for the End User Agreement:

www.umlib.nl/taverne-license

Take down policy

If you believe that this document breaches copyright please contact us at:

repository@maastrichtuniversity.nl

providing details and we will investigate your claim.
}

Copyright and moral rights for the publications made accessible in the public portal are retained by the authors and/or other copyright owners and it is a condition of accessing publications that users recognise and abide by the legal requirements associated with these

- Users may download and print one copy of any publication from the public portal for the purpose of private study or research.

- You may not further distribute the material or use it for any profit-making activity or commercial gain

If the publication is distributed under the terms of Article $25 \mathrm{fa}$ of the Dutch Copyright Act, indicated by the "Taverne" license above, 


\title{
Efficacy of amantadine on behavioural problems due to acquired brain injury: A systematic review
}

\author{
B.J. Ter Mors ${ }^{a}$, A.P.M. Backx ${ }^{b}$, P. Spauwen ${ }^{a}$, R.W.H.M. Ponds ${ }^{b, c}$, P.N. Van Harten ${ }^{d, e}$, and C.M. Van Heugten $\mathbb{0}^{c, d, f}$
}

aGGZ Oost-Brabant, Brain Injury Department Huize Padua, Boekel, The Netherlands; b Adelante Rehabilitation Centre, Hoensbroek, The Netherlands; 'Limburg Brain Injury Center, Maastricht, The Netherlands; dSchool for Mental Health and Neuroscience, Maastricht University Medical Centre, Maastricht, The Netherlands; ${ }^{e}$ GGz Centraal, Amersfoort, The Netherlands; fDepartment of Neuropsychology and Psychopharmacology, Maastricht University, Maastricht, The Netherlands

\begin{abstract}
Objectives: To systematically review literature on efficacy of amantadine on behavior (irritability/ aggression/agitation, emotional lability, apathy, impairment of executive functioning), participation, quality-of-life (QoL), and safety, in patients with acquired brain injury (ABI). Amantadine is widely used clinically, so comprehensive information on efficacy, participation, QoL and safety is relevant.

Methods: We used PRISMA Guidelines. We searched PubMed/EMBASE/CINAHL (last search 28-8-2018)

Two independent reviewers performed selection and data-extraction. Quality of studies was assessed, using CONSORT and Quality Assessment Tool for Quantitative Studies (QATFQS).

Results: Eleven out of 500 studies were included. Of five RCTs, two reported significant effects on irritability/aggression, and one no effect. One RCT on cognition no effect. One prospective cohort study showed a significant effect on executive functioning. One retrospective study was inconclusive. One single-case experimental design (SCED) study reported significant effect on apathy and three casereports indicated effects on behavior. QoL and societal participation were not measured. No safety issues emerged.

Conclusion: Amantadine may be efficacious on irritability and aggression after ABI. Amantadine is a safe drug in the presence of adequate creatinine clearance. Future studies should use designs, suitable for the heterogeneous $A B I$ population, like randomized SCEDs, and should include the effect on societal participation and QoL.
\end{abstract}

\section{ARTICLE HISTORY}

Received 30 May 2018

Revised 28 February 2019

Accepted 9 April 2019

\section{KEYWORDS}

Acquired-brain-injury;

amantadine; behaviour

\section{Introduction}

Acquired brain injury (ABI), including traumatic brain injury (TBI), Cerebro vascular accidents (CVA), infections, hydrocephalus, hypoxia, and brain tumours, is a highly prevalent condition with functional impediment in several domains: physical, cognitive, emotional, behavioural and social (1). Frontal lesions in particular often lead to behavioural consequences (2), such as apathy, agitation and aggression, emotional lability and impairment of executive functioning. Studies have reported behavioural problems in up to $40 \%$ of cases at 6 to 12 months post injury (3-7). In a long-term study, Azouvi et al. (8) showed aggressive behaviour in $20 \%$ of individuals at 5 years post injury. These behavioural consequences can have a severe negative impact on outcomes such as independent living, return to work, quality of life, and caregiver functioning $(9,10)$. Adequate assessment and treatment of these problems are therefore necessary to reduce this impact.

Medication is one of the treatment options for behavioural problems after ABI. However, the efficacy and safety of using medication to treat the behavioural consequences of brain injury has not been well established in the literature.

Amantadine is a dopamine agonist and $\mathrm{N}$-methylD-aspartate (NMDA)-receptor antagonist. It was initially used as an anti-viral agent for influenza- $\mathrm{A}$, and later as an anti-Parkinson agent, but has now been shown to be ineffective for both indications $(11,12)$.

Arciniegas et al. reported amantadine as having an effect on emotional lability and/or irritability, agitation or aggression, apathy and cognition in individuals with traumatic brain injury (TBI) based on their non-systematic review of effect studies (13). Furthermore, several reviews have covered pharmacological interventions for behavioural sequelae of $\mathrm{ABI}$, but without being able to draw conclusions about the effectiveness and safety of amantadine (14-19,21).

As to safety, some studies reported no side effects while in others some seizures or hypomania occurred, but it was unclear whether this was due to amantadine. In one case report, deterioration and impulsivity, dysarthria and ataxia, possibly linked to amantadine, were reported (20). More recently, in 2015, Sami et al. (22) undertook a systematic review of randomized controlled trials (RCTs) to evaluate the effect of amantadine and other drugs on apathy, psychomotor retardation, behavioural management and cognitive function. They found one high quality RCT that demonstrated a positive effect of amantadine on irritability (23). They did not include the occurrence of side effects in their review. 
In summary, none of these reviews has been conclusive about the efficacy of amantadine for the treatment of behavioural problems after ABI. However, the reviews looking into safety appear to agree on the relative safety of amantadine when used to treat $\mathrm{ABI}$ sequelae.

In addition to the relevant reviews to date, in the present review we have addressed the full spectrum of behavioral consequences, safety and improvement in societal participation and/or quality of life following amantadine treatment. Since ABI has a severe impact on participation and as such on perceived quality of life, a treatment should positively impact these domains of functioning, as well as alleviate distress for the individual and caregivers.

The underlying hypothesis of this systematic review was that amantadine is an efficacious and safe drug for the treatment of behavioural consequences of ABI. More specifically, we hypothesized that amantadine 1) reduces irritability/ aggression/agitation, apathy, emotional lability, and/or impairment of executive functioning and 2) increases societal participation and quality-of-life (QoL) and 3) is safe to use after ABI.

\section{Methods}

\section{Selection of studies}

We performed this review according to the Preferred Reporting Items for Systematic Reviews and Meta-Analyses (PRISMA) Guidelines (24). The review was not registered.

\section{Eligibility criteria}

Articles were included, searching from 1960 to August 28th 2018, using the following inclusion criteria (see Table 1):

1. treatment evaluation studies (Randomized Controlled Trials (RCT), Controlled Clinical Trials (CCT), pretestposttest designs, single case designs, case reports, open label group design, retrospective intervention studies, 2. published in peer-reviewed journals, 3. published in English, Dutch, German or French, 4. studies included participants with stroke or other cerebrovascular accidents, traumatic brain injury, tumour, encephalitis, meningitis, hydrocephalus or hypoxia, 5. participants had to be 18 years or older, 6. participants were treated with amantadine, 7 . outcome was measured with standardized outcome measurements, and 8 . outcome was measured on aggression/agitation, apathy, emotional lability, and/or impairment of executive functioning.

We excluded (see Table 1) studies on neurodegenerative diseases (dementia, Parkinson's disease, Huntington's disease or multiple sclerosis), studies on disorders of consciousness, and theoretical papers.

We checked the included articles for all referrals to safety, adverse events and adverse effects.

\section{Information resources}

A systematic review was performed using the following scientific databases: PubMed, EMBASE, and CINAHL, searching from 1960 to August 28th 2018.

\section{Search}

The databases were searched on the following categories or combinations of categories: 'population', 'behavioural problems (irritability/aggression/agitation, emotional lability, apathy, impairment of executive functioning)', 'participation', 'quality of life' and 'amantadine'.

The full search strategy is shown in Appendix A. For the search, individual keywords for above mentioned categories were entered separately in the MeSH and Emtree thesaurus to find the appropriate index terms used in the electronic databases.

\section{Study selection}

Studies were screened independently by two reviewers (BtM and $\mathrm{AB}$ ), based on title and abstract. If a decision about inclusion could not be made on title and abstract or when abstracts were not available, the full text was used. In case of disagreement about inclusion on the basis of title/abstract, the full text was used for deliberation.

If consensus could not be reached, a third reviewer was available. To find additional records (cross referencing), we manually searched the references of included articles and of the reviews relevant to the topic of this review. If the full text was untraceable, we contacted the author and requested the full record. Data extraction and quality assessment was done independently by two reviewers (BtM and $\mathrm{AB}$ ) and discussed to reach consensus afterwards.

\section{Data extraction}

We extracted data on the study (design, outcome domains and measurements, follow up duration, target behaviour, outcome and safety), participants (inclusion and exclusion criteria, number of participants, sex, age, aetiology, severity, time since injury), and intervention characteristics (amantadine dose, in- or outpatients, co-interventions).

Concerning treatment outcome we gathered data on effect on aggression/agitation/irritability, apathy, emotional/affect lability, impairment of executive functioning, participation and QoL. In addition, we checked the articles for QoL indices.

Regarding safety, we extracted data wherever the text mentioned side effects, adverse events or adverse effects.

\section{Quality assessment}

To assess the quality of the RCTs, we used the Consolidated Standards of Reporting Trials (CONSORT) criteria. Quality was indicated by the percentage of items reported in the articles (25). To assess risk bias of these RCTs, we used the Quality Assessment Tool for Quantitative Studies (QATFQS) from the Effective Public Health Practice Project, 2007 (EPHPP) (26). This is one of the methods for risk bias assessment recommended by the CONSORT. The method includes ratings of eight domains to be judged regarding the extent of bias that is present. One randomized single case experimental design (SCED) study was assessed using the SCED Scale by Tate (27). 
Table 1. Inclusion criteria for the selection of publications

\begin{tabular}{|c|c|}
\hline & Inclusion criteria \\
\hline Participants & acquired brain injury (TBI ${ }^{1}, \mathrm{CVA}^{2}$, tumor, encephalitis, meningitis, hydrocephalus or hypoxia) \\
\hline Treatment & amantadine \\
\hline Outcome & behavioural problems, participation, societal participation or quality of life as measured by standardized outcome measures, and safety \\
\hline Study type & treatment evaluation studies ( $\mathrm{RCT}^{3}, \mathrm{CCT}^{4}$, pre-test post-test, single case, open label group design, retrospective intervention study) \\
\hline Type of publication & peer-reviewed journals \\
\hline Year of publication & $1960-28-8-2018$ \\
\hline Language & English, German, Dutch, French \\
\hline Age of participants & adults (18 and over) \\
\hline Exclusion & $\begin{array}{l}\text { neurodegenerative diseases, disorders of consciousness, } \\
\text { outcome obscured by possible effect of other interventions, } \\
\text { reviews, theoretical papers }\end{array}$ \\
\hline
\end{tabular}

${ }^{1}$ TBI: traumatic brain injury. ${ }^{2}$ CVA: Cerebro Vascular accident, ${ }^{3}$ Randomized Controlled trial, ${ }^{4}$ Controlled Clinical trial

In the results paragraph these studies are described in more detail.

The results of non-RCT/non-randomized SCED studies are summarized (see Table 4). We did not perform a quality assessment on these studies.

\section{Results}

\section{Study selection}

The study selection is outlined in Figure 1. Of the 605 records found, 500 remained after excluding duplicates.

We excluded 470 records based on title and abstract, nine articles remained untraceable, and the full text of 23 articles was assessed for eligibility based on the in- and exclusion criteria. This includes two articles found by manually searching in the references of 44 excluded reviews.

Eleven articles were selected for this review. We manually searched the references of the included articles but found no additional articles. All selected articles were published in English.

\section{Study characteristics}

(See Table 2)

\section{Methods}

We found five RCTs Hammond's 4 studies RCT $(23,28-30)$ and Schneiders' study (31) and one randomized SCED (32). In addition we found three case reports/series (33-35), one prospective cohort intervention study (36), and one retrospective intervention study (37).

Regarding outcome measures, four studies reported on irritability/aggression/anger, two reported on apathy, two reported on behaviour in general and six reported on cognitive (executive) functioning. The follow-up period ranged from 28 days to one year. All but one study (29) included side effects as an outcome measure.

\section{Participants}

Most studies described small samples, with numbers varying from one to 168. All participants in the RCTs, the SCED, the prospective cohort study, one case report and one retrospective intervention study were individuals with TBI (a total of 514). The two case series were of mixed aetiologies (traumatic brain injury (TBI), vascular and infectious disorders; a total of 19 participants). One case report concerned a individual with brain injury due to hypoxia. In the individuals with TBI, injury severity ranged from mild to severe. All included participants, except in the retrospective intervention study, were in the chronic phase of their illness, defined as $>6$ months post-injury. Inpatients and outpatients were both included. The mean age was 40 with a range of 16-75 years. In the included studies, $43 \%$ to $77 \%$ of participants were male. Inclusion and exclusion criteria are shown in Tables 2 and 3.

Intervention. The dosage of amantadine ranged from 200 to $400 \mathrm{mgs}$ daily. In one case report (35) amantadine was combined with levodopa/carbidopa. In the other studies pharmacological co-interventions were kept stable during the study.

\section{Outcome}

Table 3 (RCTs and randomized SCED) and Table 4 (nonRCTs) show a summary of the results.

\section{Aggression/agitation/irritability/anger}

Hammond et al. (23) used the Neuro Psychiatric Inventory (NPI)-Irritability (I) and Aggression (A) domains. They reported that $81 \%$ of the participants in the amantadine group improved by at least three points on the NPI-I, compared with $44 \%$ in the placebo group (statistically significant). They found no between-group differences in change in aggression (NPI-A) when including all participants. When the individuals with baseline NPI-A scores of 0-2 (absent/ mild aggression) were excluded, there was a significant difference in favour of the intervention group $(p=.046)$. In the multisite replication on irritability only (28), the positive effect of amantadine versus placebo on irritability could not be confirmed. They found large improvement on irritability in both the intervention group and the control group. However, no statistically significant differences were noted between the groups for any of the observer NPI-I ratings at either followup interval.

Participant ratings were not statistically significant after correction for multiple comparisons.

Clinical ratings, however, reveal greater improvement for the amantadine group at day $60(p=.0354)$.

There were no differences between groups in side effect frequency, number or severity.

In their 2015 article Hammond et al (28). reported a metaanalysis on the results of these two studies, which revealed 

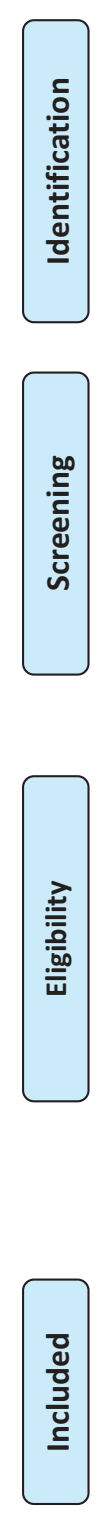

Figure 1. From: Moher D, Liberati A, Tetzlaff J, Altman DG, The PRISMA Group (2009). Preferred Reporting Items for Systematic Reviews and Meta-Analyses: The PRISMA Statement. PLoS Med 6(7): e1000097. doi:10.1371/journal.pmed1000097

a small, non-significant effect on irritability favouring amantadine over placebo.

Hammond's overall conclusion from the 2014 and 2015 $(23,28)$ publications is that: "The findings of improvement in irritability in the amantadine and the placebo group, as well as the significant improvement from clinician and participant perspectives may warrant further investigation."

A more recent RCT of Hammond et al. on the effect of amantadine on aggression and anger (29) in patients with chronic TBI showed that differences between the amantadine group and the placebo group in participant-rated day 60 NPI-A Most Problematic (adjusted $P=.0118$ ) and NPI-A Distress (adjusted $P=.0118$ ) were statistically significant, but STAXI-2 (anger) differences were not significant after adjustment for multiple comparisons.
So, amantadine $100 \mathrm{mg}$ twice daily appears to be beneficial in decreasing aggression from the perspective of the individual with TBI, but not from the observer point of view.

No beneficial impact on anger was found.

Schneider used standardized neuropsychological testing and the Neurobehavioral Rating Scale (NRS) and reported a main effect of time, but no difference between the amantadine group and the placebo group regarding disinhibition and agitation (31).

\section{Apathy}

Van Reekum performed a randomized SCED, using a selfdeveloped behavioural inventory tailored to the individual, ranking from 0 (no initiation) to 6 (perfect initiation) to score initiation of various activities for an individual with a-motivation, apathy and lack of initiation (32). The average 
Table 2. characteristics of included studies.

\begin{tabular}{|c|c|c|c|c|c|c|c|}
\hline Authors & Type study & Intervention & $\begin{array}{l}\text { Sample } \\
\text { size }\end{array}$ & $\begin{array}{l}\text { Mean age, } \\
\text { sex }\end{array}$ & Brain injury* & Outcome & Follow up \\
\hline $\begin{array}{l}\text { Hammond } \\
\text { FM, } \\
\text { et al. } \\
2018\end{array}$ & $\begin{array}{l}\text { Parallel-group, } \\
\text { randomized, double- } \\
\text { blind, placebo- } \\
\text { controlled } \\
\text { multicentre trial }\end{array}$ & $\begin{array}{l}\text { Amantadine } 100 \mathrm{mgs} \\
\text { bid (or placebo) }\end{array}$ & $\mathrm{N}=119$ & $38,6 \pm 12.4$ & $\begin{array}{l}\text { TBI }>6 \text { moths } \\
\text { post injury. } \\
\text { GCS }<13 \text {, } \\
\text { PTA }>24 h \text {, LOC } \\
\text { through TBI }\end{array}$ & $\begin{array}{l}\text { Neuropsychologic battery with well } \\
\text { established psychometric qualities testing } \\
\text { learning/memory, attention and executive } \\
\text { function at day } 28 \text { and day } 60 \text {. }\end{array}$ & 60 days \\
\hline $\begin{array}{l}\text { Hammond } \\
\text { FM, } \\
\text { et al, } \\
2017\end{array}$ & $\begin{array}{l}\text { Parallel-group, } \\
\text { randomized, double- } \\
\text { blind, placebo- } \\
\text { controlled } \\
\text { multicentre trial }\end{array}$ & $\begin{array}{l}\text { Amantadine } 100 \text { mgs } \\
\text { bid (or placebo) }\end{array}$ & $\mathrm{N}=118$ & & $\begin{array}{l}\text { Chronic, non- } \\
\text { penetrating, } \\
\text { moderate/ } \\
\text { severe, } \\
\text { traumatic BI }\end{array}$ & $\begin{array}{l}\text { NPI-A, most problematic } \\
\text { NPI-A distress, participant and observer } \\
\text { Staxi-2 participant and observer }\end{array}$ & 60 days \\
\hline $\begin{array}{l}\text { Hammond } \\
\text { FM, } \\
\text { et al., } \\
2015 .\end{array}$ & $\begin{array}{l}\text { Parallel-group, } \\
\text { randomized, double- } \\
\text { blind, placebo- } \\
\text { controlled } \\
\text { multicentre trial }\end{array}$ & $\begin{array}{l}\text { Amantadine } 100 \text { mgs } \\
\text { bid (or placebo) }\end{array}$ & $N=168$ & $\begin{array}{l}39 \text { years, } \\
77 \% \text { male }\end{array}$ & $\begin{array}{l}\text { Chronic, non- } \\
\text { penetrating, } \\
\text { moderate/ } \\
\text { severe, } \\
\text { traumatic BI }\end{array}$ & $\begin{array}{l}\text { Reduction of irritability frequency, severity, } \\
\text { and distress as measured by the } \\
\text { Neuropsychiatric Inventory Irritability (NPI-I) } \\
\text { Most problematic item (primary outcome), } \\
\mathrm{NPI} \text {-I most aberrant item, NPI-I distress, } \\
\text { at day } 28 \text { (primary endpoint) and day } 60 \text { by } \\
\text { observer (primary outcome) and participant. } \\
\text { Global impression of change by observers } \\
\text { and participants. Clinical global impression } \\
\text { by physician. Glasgow Outcome Scale - } \\
\text { Extended. Beck Depression Inventory II. }\end{array}$ & 60 days \\
\hline $\begin{array}{l}\text { Hammond } \\
\text { FM, } \\
\text { et al., } \\
2014 .\end{array}$ & $\begin{array}{l}\text { Parallel-group, } \\
\text { randomized, double- } \\
\text { blind, placebo- } \\
\text { controlled trial }\end{array}$ & $\begin{array}{l}\text { Amantadine } 100 \text { mgs } \\
\text { bid (or placebo) }\end{array}$ & $N=76$ & $\begin{array}{l}38 \text { years } \\
62 \% \text { male }\end{array}$ & $\begin{array}{c}\text { Chronic, closed, } \\
\text { traumatic Bl }\end{array}$ & $\begin{array}{l}\text { Reduction of irritability frequency (primary } \\
\text { outcome), aggression, reduction of observer } \\
\text { distress over irritability, and frequency/ } \\
\text { severity and observer distress over } \\
\text { aggression among individuals with TBI as } \\
\text { measured by NPI-I, NPI-A, NPI-Distress } \\
\text { score. Beck Depression Inventory-II, brief } \\
\text { symptom inventory, Global mental health } \\
\text { scale, fatigue impact scale }\end{array}$ & 28 days \\
\hline $\begin{array}{l}\text { Schneider } \\
\text { WN, } \\
\text { et al., } \\
1999 .\end{array}$ & $\begin{array}{l}\text { Prospective, } \\
\text { randomized, double- } \\
\text { blind, placebo- } \\
\text { controlled, crossover } \\
\text { design }\end{array}$ & $\begin{array}{l}\text { Amantadine } 50 \text { to } 150 \\
\text { mgs bid (or placebo) }\end{array}$ & $\mathrm{N}=10$ & $\begin{array}{l}31 \text { years } \\
70 \% \text { male }\end{array}$ & $\begin{array}{c}\text { Closed, } \\
\text { traumatic, } \\
\text { moderate/severe } \\
\mathrm{BI}\end{array}$ & $\begin{array}{l}\text { Orientation, attention, executive function, } \\
\text { memory, orientation, behavior, and } \\
\text { a composite variable as measured by } \\
\text { standardized neuropsychological testing } \\
\text { and the Neurobehavioural Rating Scale. }\end{array}$ & 6 weeks \\
\hline $\begin{array}{l}\text { Reekum } \\
\text { R van, } \\
\text { et al., } \\
1995 .\end{array}$ & $\begin{array}{l}\text { Single case } \\
\text { experimental design }\end{array}$ & $\begin{array}{l}\text { Amantadine } 100 \mathrm{mgs} \\
\text { tid or placebo }\end{array}$ & $\mathrm{N}=1$ & $\begin{array}{l}42 \text { years, } \\
\text { male }\end{array}$ & $\begin{array}{c}\text { Chronic severe } \\
\text { traumatic Bl }\end{array}$ & $\begin{array}{l}\text { Score on a behaviour inventory targeted to } \\
\text { the subject, with each item ranked from } 0 \\
\text { (no initiation) to } 6 \text { (perfect initiation). Side } \\
\text { effects including aggression, difficult } \\
\text { behaviour and psychosis }\end{array}$ & 8 weeks \\
\hline $\begin{array}{l}\text { Kraus MF, } \\
\text { et al., } \\
2005 .\end{array}$ & $\begin{array}{l}\text { Prospective cohort, } \\
\text { intervention study, } \\
\text { open-label group } \\
\text { design }\end{array}$ & $\begin{array}{l}\text { Amantadine } 200 \text { mgs } \\
\text { od } 100 \text { mgs bid }\end{array}$ & $N=22$ & $\begin{array}{l}36 \text { years } \\
77 \% \text { male }\end{array}$ & $\begin{array}{l}\text { Chronic, closed, } \\
\text { traumatic, mild/ } \\
\text { moderate/severe } \\
\text { BI }\end{array}$ & $\begin{array}{l}\text { Cognition (executive functions) as } \\
\text { measured by a focused neuropsychological } \\
\text { test battery dependent on intact pre-frontal } \\
\text { cortex function. Attention and memory } \\
\text { domains were secondary outcome } \\
\text { measures. }\end{array}$ & 12 weeks \\
\hline $\begin{array}{l}\text { Nickels JL, } \\
\text { et al., } \\
1994 .\end{array}$ & $\begin{array}{l}\text { Retrospective case } \\
\text { series }\end{array}$ & $\begin{array}{l}\text { Amantadine } 200-300 \\
\text { mgs daily }\end{array}$ & $\mathrm{N}=12$ & $\begin{array}{l}37 \text { years } \\
50 \% \text { male }\end{array}$ & $\begin{array}{l}\text { Acute - chronic, } \\
\text { traumatic or } \\
\text { vascular or } \\
\text { infectious BI }\end{array}$ & $\begin{array}{l}\text { Depending on subject: aggression, } \\
\text { cognitive functioning (attention measured } \\
\text { by Trail A), level of consciousness/ } \\
\text { responsiveness. Secondary outcome: side } \\
\text { effects. }\end{array}$ & unclear \\
\hline $\begin{array}{l}\text { Kraus FM, } \\
\text { et al., } \\
1997 .\end{array}$ & $\begin{array}{l}\text { Case studies (and } \\
\text { review) naturalistic } \\
\text { no on off regime. }\end{array}$ & $\begin{array}{l}\text { Amantadine } 25-400 \\
\text { mgs daily }\end{array}$ & $\mathrm{N}=7$ & $\begin{array}{l}37.5 \text { years } \\
43 \% \text { male }\end{array}$ & $\begin{array}{l}\text { Chronic } \\
\text { traumatic/ } \\
\text { infectious BI }\end{array}$ & $\begin{array}{l}\text { Neuropsychiatric evaluation results at } \\
\text { baseline and follow-up visits. } \\
\text { Neuropsychological evaluation included } \\
\text { tests selected to measure a variety of } \\
\text { cognitive functions, with particular } \\
\text { emphasis on tests sensitive to frontal lobe } \\
\text { function. }\end{array}$ & $3-6$ months \\
\hline $\begin{array}{l}\text { Arciniegas } \\
\text { DB, } \\
\text { et al., } \\
2004 .\end{array}$ & Case report $(A B A B)$ & $\begin{array}{l}\text { Amantadine } 100 \mathrm{mgs} \\
\text { bid }\end{array}$ & $\mathrm{N}=1$ & $\begin{array}{l}24 \text { years, } \\
\text { Male }\end{array}$ & $\begin{array}{l}\text { Subacute severe } \\
\text { hypoxic - } \\
\text { ischemic Bl }\end{array}$ & $\begin{array}{l}\text { Performance on the Attention Process } \\
\text { Training (APT) and Rivermead Behavioural } \\
\text { Memory Test (RBMT). Cognition tested by } \\
\text { bedside cognitive testing. Apathy assessed } \\
\text { by clinical observation }\end{array}$ & 40 weeks \\
\hline $\begin{array}{l}\text { Kraus MF, } \\
\text { et al., } \\
1997 .\end{array}$ & Case report & $\begin{array}{l}\text { Amantadine } 400 \text { mgs } \\
\text { daily at first, later } \\
\text { combined with } \\
\text { levodopa/carbi-dopa } \\
25 / 100 \text { tid }\end{array}$ & $N=1$ & $\begin{array}{l}\text { 'In her } \\
\text { fifties', } \\
\text { woman }\end{array}$ & $\begin{array}{c}\text { Chronic, severe } \\
\text { traumatic Bl }\end{array}$ & $\begin{array}{l}\text { Cognitive functions, with an emphasis on } \\
\text { frontal lobe function, measured by } \\
\text { a neuropsychological test battery and } \\
\text { clinical judgement of behaviour. }\end{array}$ & 1 year \\
\hline
\end{tabular}

if authors did not stage the duration of brain injury, we classified brain injury of 0-2 months as acute, 2- 6 months as subacute, over 6 months as chronic bid: bis in die (twice daily), tid: ter in die (three times a day) 
Table 3. Results of included RCTs.

\begin{tabular}{|c|c|c|c|c|c|}
\hline & In-exclusion criteria & Patient group & Control group & Results & Quality* \\
\hline $\begin{array}{l}\text { Hammond } \\
\text { FM, et al. } \\
2015\end{array}$ & $\begin{array}{l}\text { Inclusion: (1) } 16-75 \text { years old, } \\
\text { (2) nonpenetrating TBI at least } \\
6 \text { months before enrollment, } \\
\text { and (3) score } \geq 6 \text { on observer- } \\
\text { rated NPI-I most problematic. } \\
\text { Medical and neurological } \\
\text { stability, ability to comply with } \\
\text { the study protocol, negative } \\
\text { pregnancy test, and creatinine } \\
\text { clearance }>60 \text {. An observer with } \\
\text { adequate interaction with the } \\
\text { person with TBI to observe } \\
\text { irritability was required. } \\
\text { Exclusion: (1) inability to } \\
\text { interact and communicate, (2) } \\
\text { threat of harm to self or other, } \\
\text { (3) diagnosis of other neurologic } \\
\text { disorder, (4) schizophrenia or } \\
\text { psychosis, (5) seizure in the } \\
\text { month before enrollment, (6) } \\
\text { concomitant use of typical } \\
\text { neuroleptic agents or } \\
\text { monoamine oxidase inhibitors } \\
\text { (because of potential drug } \\
\text { interactions), (7) previous } \\
\text { adverse reaction to amantadine, } \\
\text { (8) treatment with amantadine } \\
\text { during the month before } \\
\text { enrollment, and (9) enrollment } \\
\text { in the previous single-site } \\
\text { amantadine irritability study. }\end{array}$ & $\begin{array}{l}\text { TBI was verified by record review } \\
\text { and clinician interview with } \\
\text { a requirement to meet at least } \\
\text { one of the following criteria: (1) } \\
\text { post-resuscitation Glasgow } \\
\text { Coma Scale (GCS) } \leq 13 ; \text {; }(2) \mathrm{GCS} \\
\text { Motor <6 off paralytics; (3) any } \\
\text { period of loss of consciousness } \\
\text { attributable to TBl; (4) post- } \\
\text { traumatic amnesia lasting } \geq 24 \mathrm{~h} \text {; } \\
\text { (5) neuroimaging consistent } \\
\text { with TBl; and/or (6) other } \\
\text { evidence of TBI-related focal } \\
\text { neurological findings. } \\
\text { Causes TBI: Vehicular } 65 \% \text {, Fall } \\
17 \% \text {, Assault } 8 \% \text { Sport-related } \\
2 \% \text {, Pedestrian } 5 \%, \text { Other } 2 \% \text {. * }\end{array}$ & $\begin{array}{l}\text { The amantadine and placebo } \\
\text { groups were well matched with } \\
\text { respect to baseline factors with } \\
\text { the exception of a small but } \\
\text { statistically significant } \\
\text { difference ( } p=.0492) \text { in mean } \\
\text { baseline extended Glasgow } \\
\text { Outcome Scale (GOS-E). } \\
\text { Compliance was similar. }\end{array}$ & $\begin{array}{l}\text { There were large improvements } \\
\text { in both groups; however, no } \\
\text { statistically significant } \\
\text { differences were noted between } \\
\text { the groups for any of the } \\
\text { observer NPI-I ratings at either } \\
\text { follow-up interval. } \\
\text { Participant ratings were not } \\
\text { statistically significant with } \\
\text { correction for multiple } \\
\text { comparisons. } \\
\text { Clinical ratings reveal greater } \\
\text { global improvement for the } \\
\text { amantadine group at day } 60 \\
(p=.0354) \text {. } \\
\text { No difference between groups in } \\
\text { side effects. }\end{array}$ & $\begin{array}{l}\text { CONSORT } \\
28 \\
\text { QTAQS } \\
\text { overall } \\
\text { Moderate }\end{array}$ \\
\hline $\begin{array}{l}\text { Hammond } \\
\text { FM, et al. } \\
2014\end{array}$ & $\begin{array}{l}\text { Inclusion: } 16 \text { to } 65 \text { years old, } \\
\text { closed head injury due to } \\
\text { trauma at least } 6 \text { months prior } \\
\text { to enrollment with a score } \\
\text { greater than } 2 \text { on NPI-Irritability } \\
\text { domain, medical and } \\
\text { neurological stability, the ability } \\
\text { to give informed consent and } \\
\text { comply with study protocol, } \\
\text { a negative pregnancy test, and } \\
\text { creatinine clearance greater than } \\
60 \text { mg/dL. } \\
\text { Exclusion: anticipated surgery } \\
\text { or medication change during } \\
\text { the study, diagnosis of other } \\
\text { neurologic disorder, seizure in } \\
\text { the month prior to enrollment, } \\
\text { concomitant use of neuroleptic } \\
\text { agents or monoamine oxidase } \\
\text { inhibitors, previous allergy or } \\
\text { adverse reaction to amantadine, } \\
\text { and ingestion of amantadine in } \\
\text { the month prior to study } \\
\text { enrollment. Participants were } \\
\text { required to have an observer, } \\
\text { who was willing and able to } \\
\text { observe presence of irritability. }\end{array}$ & $\begin{array}{l}\text { Causes TBI: Vehicular } 62 \% \text {, Fall } \\
12 \% \text {, Assault } 3 \% \text {, Sport-related } \\
1 \% \text {, Pedestrian } 12 \% \text {, Other } 11 \% \text {. } \\
{ }_{*}\end{array}$ & $\begin{array}{l}\text { The amantadine and placebo } \\
\text { groups were well matched with } \\
\text { respect to baseline factors. } \\
\text { Compliance was similar. }\end{array}$ & $\begin{array}{l}\text { In the amantadine group, } \\
80.56 \% \text { improved at least } 3 \\
\text { points on the NPI-I, compared } \\
\text { with } 44.44 \% \text { in the group that } \\
\text { received placebo ( } P=.0016) \text {. } \\
\text { Mean change in NPI-I was }-4.3 \\
\text { in the amantadine group and } \\
-2.6 \text { in the placebo group } \\
(P=.0085) \text {. When excluding } \\
\text { individuals with minimal to no } \\
\text { baseline aggression, mean } \\
\text { change in NPI-A was }-4.56 \text { in } \\
\text { the amantadine group and } \\
-2.46 \text { in the placebo group } \\
(P=.046) \text {. Mean changes in NPI- } \\
\text { I and NPI-A distress were not } \\
\text { statistically significant between } \\
\text { the amantadine and placebo } \\
\text { groups. Adverse event } \\
\text { occurrence did not differ } \\
\text { between the } 2 \text { groups }\end{array}$ & $\begin{array}{l}\text { CONSORT } \\
24 \\
\text { QATQS } \\
\text { overall } \\
\text { strong }\end{array}$ \\
\hline $\begin{array}{l}\text { Schneider } \\
\text { WN, } \\
\text { et al. }\end{array}$ & $\begin{array}{l}\text { Inclusion: (1) diagnosis of } \\
\text { closed head injury, (2) no prior } \\
\text { psychiatric history, (3) age } \\
\text { between } 18 \text { and } 55 \text { years, and } \\
\text { (4) deficits in attention/ } \\
\text { concentration documented per } \\
\text { neuropsychological screening. } \\
\text { Exclusion: (1) history of cardiac } \\
\text { arrhythmia, and (2) subjects } \\
\text { suspected to be pregnant. }\end{array}$ & $\begin{array}{l}\text { The subjects all had significant } \\
\text { head injury marked by loss of } \\
\text { consciousness and amnesia. } \\
\text { Most had an initial Glasgow } \\
\text { Coma Scale score below } 9 \text {. } \\
\text { Nearly all (nine of 10) subjects } \\
\text { had intracerebral haemorrhage } \\
\text { and/or cerebral contusion on CT } \\
\text { scan. Three subjects experienced } \\
\text { acute seizure. Ventriculostomies } \\
\text { were required for two of the } \\
\text { subjects. * }\end{array}$ & Not applicable; cross over study & $\begin{array}{l}\text { Repeated measures ANOVA and } \\
\text { regression analysis of slope } \\
\text { showed a main effect of time } \\
\text { without difference for } \\
\text { amantadine versus placebo. No } \\
\text { side effects mentioned. } \\
\text { Comment: } \\
\text { In addition to the } 10 \text { subjects } \\
\text { reported here, eight subjects did } \\
\text { not complete the study. }\end{array}$ & $\begin{array}{l}\text { CONSORT } \\
12 \\
\text { QATQS } \\
\text { overall } \\
\text { weak }\end{array}$ \\
\hline
\end{tabular}


Table 3. (Continued).

\begin{tabular}{|c|c|c|c|c|c|}
\hline & In-exclusion criteria & Patient group & Control group & Results & Quality* \\
\hline $\begin{array}{l}\text { Reekum, } \\
\text { R van, } \\
\text { et al. }\end{array}$ & $\begin{array}{l}\text { 42-year-old male with apathy, } \\
\text { amotivation, abulia, slowness, } \\
\text { lack of initiation, little insight } \\
\text { and perseveration due to } \\
\text { a severe traumatic brain injury. }\end{array}$ & $\begin{array}{l}\text { Single Case Experimental Design } \\
\text { (RCT) }\end{array}$ & $\begin{array}{l}\text { Single Case Experimental } \\
\text { design (RCT) }\end{array}$ & $\begin{array}{l}\text { Average effect score increased } \\
\text { from } 0.86 \text { on placebo to } 1.79 \text { on } \\
\text { amantadine. The average side } \\
\text { effect score decreased from } 0.53 \\
\text { on placebo to } 0.03 \text { on } \\
\text { amantadine }\end{array}$ & Tate $3 / 11$ \\
\hline $\begin{array}{r}\text { Hammond, } \\
\text { FM } 2018\end{array}$ & $\begin{array}{l}\text { Inclusion:Individuals were } \\
\text { eligible if } 16-75 \text { years of age, } \\
\text { sustained a nonpenetrating TBI } \\
\text { at least } 6 \text { months prior to } \\
\text { enrollment, and obtained } \\
\text { a score_>6 on observer-rated } \\
\text { Neuropsychiatric Inventory- } \\
\text { Irritability. An observer available. } \\
\text { medical and neurological } \\
\text { stability, ability to comply with } \\
\text { study protocol, negative } \\
\text { pregnancy test, and creatinine } \\
\text { clearance >60. } \\
\text { significant cognitive impairment } \\
\text { as indicated } \\
\text { by two or more cognitive test } \\
\text { scores at least } 1 \text { standard } \\
\text { deviation (SD) below normative } \\
\text { values } \\
\text { Exclusion: } 1 \text { ) unable to interact } \\
\text { and communicate; } 2 \text { ) threat of } \\
\text { harm to self or other; } 3 \text { ) history } \\
\text { of neurologic disorder, } \\
\text { schizophrenia, or psychosis; 4) } \\
\text { seizure in month preceding } \\
\text { enrollment; 5) concomitant use } \\
\text { of typical neuroleptic agents or } \\
\text { monoamine oxidase inhibitors } \\
\text { (because of potential drug } \\
\text { interactions); and 6) amantadine } \\
\text { ingested during the month } \\
\text { preceding enrollment. }\end{array}$ & $\begin{array}{l}\text { TBI was verified by record review } \\
\text { and clinician interview with } \\
\text { a requirement to meet at least } \\
\text { one of the following criteria: (1) } \\
\text { post-resuscitation Glasgow } \\
\text { Coma Scale (GCS) } \leq 13 ; \text {; }(2) \text { GCS } \\
\text { Motor <6 off paralytics; (3) any } \\
\text { period of loss of consciousness } \\
\text { attributable to TBl; (4) post- } \\
\text { traumatic amnesia lasting } \geq 24 \mathrm{~h} \text {; } \\
\text { (5) neuroimaging consistent } \\
\text { with TBl; and/or (6) other } \\
\text { evidence of TBI-related focal } \\
\text { neurological findings. } \\
\text { Causes TBI: Vehicular } 65 \% \text {, Fall } \\
17 \% \text {, Assault } 8 \% \text { Sport-related } \\
2 \% \text {, Pedestrian } 5 \% \text {, Other } 2 \% \text {. * }\end{array}$ & $\begin{array}{l}\text { Groups were well matched } \\
\text { with respect to baseline factors }\end{array}$ & $\begin{array}{l}\text { A high percentage of those in } \\
\text { the placebo group showed } \\
\text { positive change from baseline } \\
\text { to day } 28(59-65 \%) \text { compared to } \\
\text { the amantadine group (46-53\%) } \\
\text { on all CVLT measures. } \\
\text { At day } 60 \text {, no significant } \\
\text { between-group differences were } \\
\text { observed for any of the indices. } \\
\text { Amantadine was well tolerated } \\
\text { among study participants with } \\
\text { no } \\
\text { significant between-group } \\
\text { differences on withdrawals/lost } \\
\text { or adverse } \\
\text { events }\end{array}$ & $\begin{array}{l}\text { CONSORT: } \\
31 \\
\text { QATQS } \\
\text { overall } \\
\text { strong }\end{array}$ \\
\hline
\end{tabular}

${ }^{*}$ CONSORT: see Table 5

QATQS: see Table 6 
Table 4. Results of included non-randomized studies.

\begin{tabular}{|c|c|c|}
\hline Authors & Patient group & Results \\
\hline $\begin{array}{l}\text { Kraus MF, } \\
\text { et al. } \\
2005\end{array}$ & $\begin{array}{l}\text { Time out from injury (In months, mean, range) }=63.2(6-240) . \text { DSM-IV: } \\
\text { cognitive disorder NOS. Severity of TBI, mild/moderate/severe* } 6 / 6 / 10 \\
\text { (based on Glasgow Coma scale) * type of TBI closed TBI at least } 6 \text { months } \\
\text { post-injury. A sub-set of six male subjects underwent PET scanning, not } \\
\text { relevant for this review. * Patients were excluded if there was a history of } \\
\text { prior psychiatric disorder, substance abuse or any other neurologic } \\
\text { condition or medical condition that could result in cognitive changes. }\end{array}$ & $\begin{array}{l}\text { On the executive function domain score, subjects significantly improved } \\
\text { following treatment with amantadine, } \mathrm{t}(20)=-2.47, p=.02 \text {, two-tailed. } \\
\text { There were no significant differences for either the attention or the } \\
\text { memory domains. } \\
\text { Side effects included mild light-headedness and nausea, which resolved } \\
\text { after discontinuing drug, and one case of livedo reticularis. }\end{array}$ \\
\hline $\begin{array}{l}\text { Nickels JL, } \\
\text { et al. }\end{array}$ & $\begin{array}{l}9 \text { with TBI, } 2 \text { with SAH, } 1 \text { with encephalitis. Three of the patients showed } \\
\text { aggression and attention disorders, } 9 \text { had disorders of conciousness. }\end{array}$ & $\begin{array}{l}\text { Some effect in } 10 \text { subjects (in cognitive functioning, alertness, arousal, } \\
\text { agitation, anxiety and participation in therapy), no effect in } 2 \text { subjects (of } \\
\text { which one with agitation). Two of the three patienst with agitation } \\
\text { showed resolution of agitation and anxiety. Side effects: hallucinations } \\
\text { (1), hypomania (2), seizure (2), pedal oedema (1). }\end{array}$ \\
\hline $\begin{array}{l}\text { Kraus FM, } \\
\text { et al. } \\
1997\end{array}$ & At least 1 year post-injury. & $\begin{array}{l}3 \text { responders (positive effects on neuropsychiatric evaluation }+2 \text { SD } \\
\text { improvement on at least one neuropsychological test.), } 4 \text { partial } \\
\text { responders (positive effect on neuropsychiatric evaluation, but no } \\
\text { significant change in neuropsychological testing). } \\
\text { Side effects: light headedness }\end{array}$ \\
\hline $\begin{array}{l}\text { Kraus MF, } \\
\text { et al. } \\
1997\end{array}$ & $\begin{array}{l}\text { Woman in her fifties with severe TBI resulting in cognitive deficits, } \\
\text { agitation, emotional liability, impulsivity, distractibility, perseveration, } \\
\text { episodes of low mood. }\end{array}$ & $\begin{array}{l}\text { After treatment with amantadine alone, she showed improvements in } \\
\text { her general behavior, memory, attentiveness, concentration and } \\
\text { decreased impulsivity and perseveration. On repeated testing she } \\
\text { showed gains on tests sensitive to frontal functions, but no significant } \\
\text { gains in memory. Further positive response was obtained with the } \\
\text { addition of I-dopa/carbidopa. No side effects were observed, and the } \\
\text { patient maintained gains at follow-up. }\end{array}$ \\
\hline
\end{tabular}

effect score increased from 0.86 on placebo to 1.79 on amantadine. No statistical analysis was performed.

In addition, the non-RCT/SCED studies yielded positive results on behaviour (mainly aggression/agitation and apathy) $(33,34,36,37)$.

\section{Impairment of executive functioning}

In Hammond's RCT on the effect of amantadine on cognition including executive function (29) a higher percentage of participants in the placebo group showed a positive change from baseline to day 28 as compared to the amantadine group on all measures. At 60 days there was no difference between groups. So the overall conclusion was that amantadine 100 mgs twice daily does not affect cognition, including executive function, positively.

Schneider reported a main effect of time with no difference between the amantadine versus placebo-group in executive functioning, in his RCT (31).

The non-RCT/SCED studies reported positive effect on executive functioning $(33,34,36)$.

\section{Quality of life - societal participation}

None of the included studies measured quality of life or societal participation.

\section{Safety}

All but one study (31) addressed adverse effects and/or side effects. Hammond $(23,28)$ found that adverse event occurrence did not differ between the amantadine and the placebo group. Side effects/adverse effects reported a total of 177 times in the included studies (see Appendix B). In the supplementary data on adverse effects, Hammond (28) reported no significant difference in emergence of executive function impairment (considered as an adverse effect) and agitation/aggression and irritability (considered as an adverse effect) between the placebo and amantadine groups (28). Van Reekum defined difficult behaviour, aggression and psychosis as potential side effects (32). In that study the average side effect score decreased from 0.53 on placebo to 0.03 on amantadine. This decrease in side effect score indicates an improvement in behaviour. However, there was also an increase in socially inappropriate behaviour (giggling). Two case reports $(34,35)$ described the use of amantadine without side effects/adverse effects. There is no evidence from this review that higher doses gave more adverse events. For example Van Reekum (32) used 100 mgs 3 tid and reports a decrease of side effects on amantadine versus placebo, and Hammond $(23,28-30)$ found no difference between the amantadine group and placebo on 100 mgs bid.

\section{Appraisal}

Of the five included RCTs, the Hammond studies (23,28-30) showed an overall strong quality and the 1999 study by 
Table 5. CONSORT 2010 checklists RCTs.

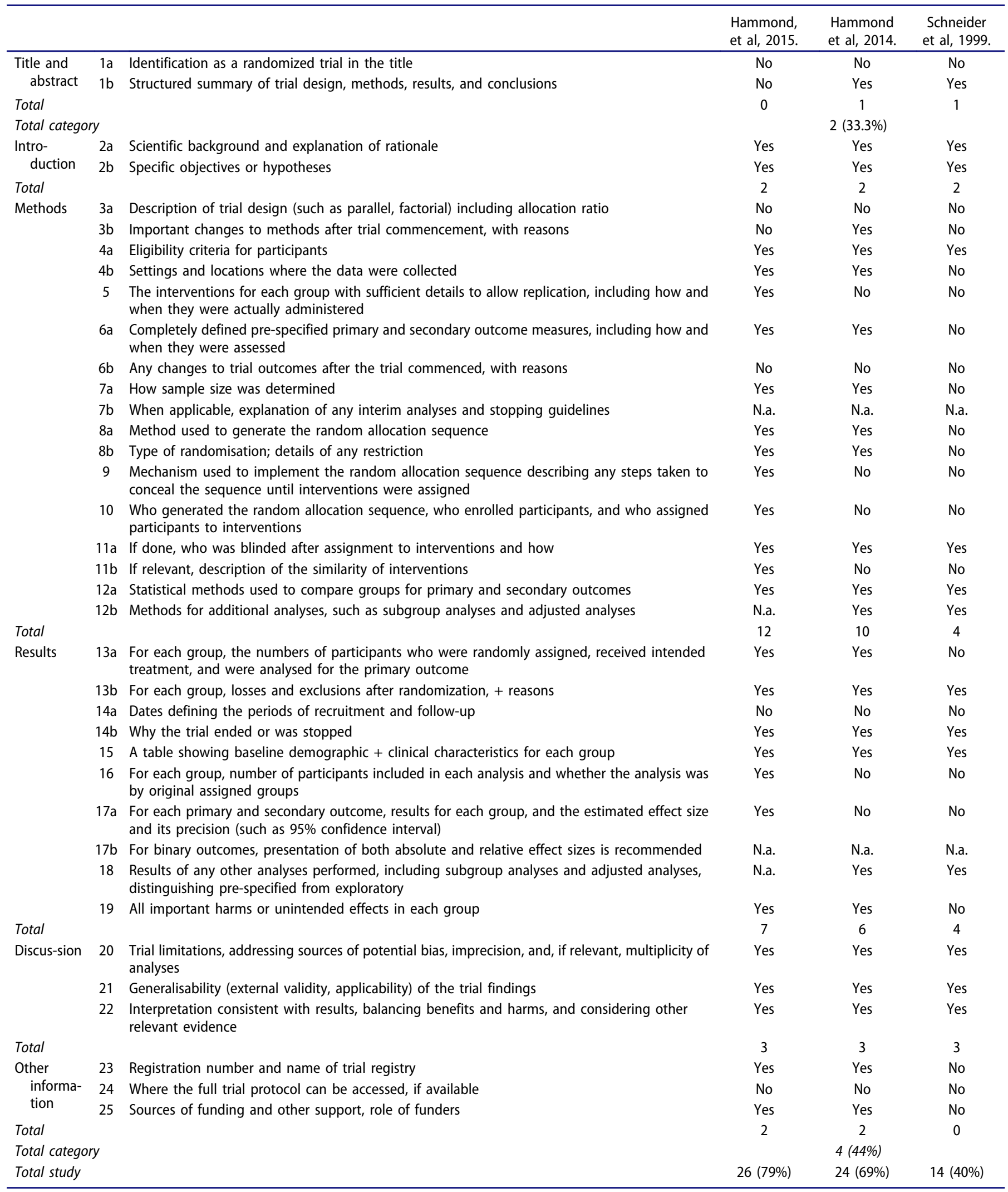


Table 5. (Continued).

\begin{tabular}{|c|c|c|c|c|}
\hline & & & $\begin{array}{l}\text { Hammond, } \\
\text { et al, } 2018 .\end{array}$ & $\begin{array}{l}\text { Hammond } \\
\text { et al, } 2017 .\end{array}$ \\
\hline \multirow{2}{*}{$\begin{array}{l}\text { Title and } \\
\text { abstract }\end{array}$} & $1 \mathrm{a}$ & Identification as a randomized trial in the title & yes & No \\
\hline & $1 \mathrm{~b}$ & Structured summary of trial design, methods, results, and conclusions & yes & Yes \\
\hline \multirow{2}{*}{$\begin{array}{l}\text { Intro- } \\
\text { duction }\end{array}$} & $2 a$ & Scientific background and explanation of rationale & Yes & Yes \\
\hline & $2 \mathrm{~b}$ & Specific objectives or hypotheses & Yes & Yes \\
\hline Total & & & 2 & 2 \\
\hline \multirow{13}{*}{ Methods } & $3 b$ & Important changes to methods after trial commencement, with reasons & yes & no \\
\hline & $4 a$ & Eligibility criteria for participants & Yes & Yes \\
\hline & $4 \mathrm{~b}$ & Settings and locations where the data were collected & Yes & Yes \\
\hline & 5 & $\begin{array}{l}\text { The interventions for each group with sufficient details to allow replication, including how and when they } \\
\text { were actually administered }\end{array}$ & Yes & Yes \\
\hline & $6 a$ & $\begin{array}{l}\text { Completely defined pre-specified primary and secondary outcome measures, including how and when } \\
\text { they were assessed }\end{array}$ & Yes & Yes \\
\hline & $8 a$ & Method used to generate the random allocation sequence & Yes & Yes \\
\hline & $8 b$ & Type of randomisation; details of any restriction & Yes & Yes \\
\hline & 9 & $\begin{array}{l}\text { Mechanism used to implement the random allocation sequence describing any steps taken to conceal the } \\
\text { sequence until interventions were assigned }\end{array}$ & Yes & yes \\
\hline & 10 & $\begin{array}{l}\text { Who generated the random allocation sequence, who enrolled participants, and who assigned participants } \\
\text { to interventions }\end{array}$ & Yes & No \\
\hline & $11 \mathrm{a}$ & If done, who was blinded after assignment to interventions and how & Yes & Yes \\
\hline & $11 \mathrm{~b}$ & If relevant, description of the similarity of interventions & no & No \\
\hline & $12 a$ & Statistical methods used to compare groups for primary and secondary outcomes & Yes & Yes \\
\hline & $12 \mathrm{~b}$ & Methods for additional analyses, such as subgroup analyses and adjusted analyses & N.a. & Yes \\
\hline Total & & & 13 & 11 \\
\hline \multirow[t]{4}{*}{ Results } & $13 a$ & $\begin{array}{l}\text { For each group, the numbers of participants who were randomly assigned, received intended treatment, } \\
\text { and were analysed for the primary outcome }\end{array}$ & Yes & Yes \\
\hline & $17 \mathrm{~b}$ & For binary outcomes, presentation of both absolute and relative effect sizes is recommended & N.a. & N.a. \\
\hline & 18 & $\begin{array}{l}\text { Results of any other analyses performed, including subgroup analyses and adjusted analyses, } \\
\text { distinguishing pre-specified from exploratory }\end{array}$ & N.a. & Yes \\
\hline & 19 & All important harms or unintended effects in each group & Yes & Yes \\
\hline Total & & & 8 & 6 \\
\hline \multirow[t]{3}{*}{ Discus-sion } & 20 & Trial limitations, addressing sources of potential bias, imprecision, and, if relevant, multiplicity of analyses & Yes & Yes \\
\hline & 21 & Generalisability (external validity, applicability) of the trial findings & Yes & Yes \\
\hline & 22 & $\begin{array}{l}\text { Interpretation consistent with results, balancing benefits and harms, and considering other relevant } \\
\text { evidence }\end{array}$ & Yes & Yes \\
\hline Total & & & 3 & 3 \\
\hline \multirow{3}{*}{$\begin{array}{l}\text { Other } \\
\text { informa- } \\
\text { tion }\end{array}$} & 23 & Registration number and name of trial registry & Yes & Yes \\
\hline & 24 & Where the full trial protocol can be accessed, if available & yes & No \\
\hline & 25 & Sources of funding and other support, role of funders & Yes & Yes \\
\hline Total & & & 3 & 2 \\
\hline Total study & & & $31(83 \%)$ & $25(68 \%)$ \\
\hline
\end{tabular}

Schneider (31) showed an overall weak quality. The results of the quality assessment are shown in Table 5 for CONSORT and Table 6 for QATFQS. The randomized SCED by van Reekum (32) fulfilled only three criteria out of 11 on de SCED quality scale (27)

\section{Discussion}

This systematic review shows that there is some evidence of the efficacy of amantadine for the reduction of irritability and aggression in individuals with TBI. This conclusion is mainly based on the publications (RCTs) of Hammond et al $(23,28-30)$. 
Table 6. Quality assessment tool for quantitative studies*.

\begin{tabular}{llllll}
\hline & Hammond, et al, 2015. & Hammond et al, 2014. & Schneider et al, 1999. & Hammond et al 2018 & Hammond et al 2017 \\
\hline Selection bias & Weak & Moderate & Weak & Moderate & Moderate \\
Study design & Strong & Strong & Weak & Strong & Strong \\
Confounders & Strong & Strong & Weak & Strong & Strong \\
Blinding & Moderate & Moderate & Moderate & Moderate & Moderate \\
Data collection method & Strong & Strong & Strong & Strong & Strong \\
Withdrawals/dropouts & Strong & Strong & Weak & Strong & Strong \\
Final decision & Moderate & Strong & Weak & Strong & Strong \\
\hline
\end{tabular}

* Quality Assessment Tool For Quantitative Studies by Effective Public Health Practice Project (26)

After completing the multisite replication (28) of their 2014 RCT (23) on irritability and aggression and combining the results, Hammond et al. conclude that, from the perspective of clinicians, amantadine $100 \mathrm{mg}$ every morning and noon may reduce irritability and the perceived distress associated with irritability, but that there was no change in the primary outcome which is improvement from the observer point of view. Their 2017 study (29) confirms that amantadine may have a positive effect on aggression from the participant and clinician point of view, but not on anger.

No effect on executive function could be established

These outcomes are supported by case reports and case series indicating potential positive effects on behavioural outcome measures, amongst others irritability and aggression.

Of five RCTs and one randomized SCED, four were of high quality. Moreover, participation and quality of life have not been studied as yet.

Of clinical importance is that amantadine seems to be a safe drug for individuals with $\mathrm{ABI}$, according to the studies that reported side effects, given adequate creatinine clearance.

The first hypothesis tested in our study concerns the efficacy of amantadine for reducing irritability/aggression/agitation, apathy, emotional lability and/or impairment in executive functioning due to ABI. We found some evidence of efficacy on irritability and aggression, but no evidence of efficacy in other behavioural domains.

The second hypothesis, that amantadine leads to increased participation and QoL, could not be tested due to a lack of data about this topic. None of the included studies addressed QoL or societal participation specifically, and none of the included articles mentioned QoL and participation.

In any case, QoL and societal participation should be included in future studies, once efficacy and safety have been established.

The third hypothesis, on the safety of amantadine, could be tested. Based on the results of this review, amantadine appears to be a safe drug for use in individuals with brain injury given adequate creatinine clearance.

This review yielded no side effects or adverse events that were not previously reported from the use of amantadine in other populations. Moreover, four high quality RCTs showed no difference in the occurrence of side effects between the amantadine group and the placebo group.

The more severe side effects like hypomania (2 out of 177), hallucinations ( 3 out of 177) and seizures (4 out of 177) were infrequent. Amantadine therefore seems to be safe in patients after brain injury, assuming the usual contraindications and precautions, (pregnancy, low creatinine clearance, refractory epilepsy, caution in the presence of congestive heart failure, no abrupt discontinuation). Note that the terms side effects, adverse effects and adverse events are terms that were used without definition in the articles in this review. We included all terms referring to safety, regardless of the definition.

\section{Implications for further research}

To explain the marked reduction in irritability and aggression in both the placebo group and the amantadine group at six months post-injury, Hammond et al. (28) suggested that nonspecific factors had a larger effect on behaviour, making the relatively small potential response to amantadine invisible. Being part of the investigation and the inherent positive attention was one of the nonspecific factors mentioned. As a solution to that problem they suggest three options: 1) a group RCT with a placebo run-in design where all subjects receive placebo after baseline and before randomization, 2) a Sequential Parallel Comparison design, which is an adaptive design that allows for re-randomization of specific placebo subjects ("non-responders") from an early stage to placebo or treatment in a subsequent stage of the trial, or 3) mega-studies : multicenter trials with $>1000$ subjects to increase power

All of these options could detect even the slightest of effects, even those with little or no clinical relevance. With this in mind, and considering the heterogeneity of the brain injury population, the usefulness of adapting the group design as Hammond suggested is doubtful.

Another option is to try to avoid the methodological problem by performing randomized Single Case Experimental Design (N-of-1 design) trials.

Single case experimental designs (SCED) are considered qualitatively strong and are frequently used in the field of brain injury because of the heterogeneity of the population $(38,39)$.

By performing single case, placebo controlled, double blind, randomized trials in direct single site and systematic multisite replication (41), responders and non-responders can be identified, i.e. the prototypical responder. By studying patient characteristics and the research process accurately, hypotheses can be developed as to why good quality group RCTs show significant improvement in both the amantadine group and the placebo group. Generality of findings require direct and systematic replication (41). If all $\mathrm{N}$-of- 1 trials in a series are accounted for combining data from multiple $\mathrm{N}$-of1 randomized placebo controlled trials with data from RCTs, using Bayesian hierarchical models, estimates of effect on a population level can be produced (40-43). Both the 
hypothetical prototypical responder and the hypotheses on methodology can create possibilities for newly designed group RCTs, possibly yielding more clear-cut results.

Furthermore, the included studies used different outcome measures and different measurement tools. To ensure comparability, the use of universal standard outcome measurements and tools is advisable. For example, common data elements are currently available from the National Institute of Neurological Disorders and Stroke (NINDS). Due to the heterogeneity in the patient population, tailored individual outcome measures, which are especially useful in N-of- 1 studies, could also be used alongside standard measurements. This may clarify the relationship between intervention and behavioural change. In particular, the combination of the standard outcome measurements and tools with an individualized outcome measurement would make identifying and analysing this relationship more feasible. Behavioural changes not covered and detected by the standard measurements could be identified by the tailored measurement.

\section{Limitations of this review}

One limitation of this review is the incompleteness of study collection. We were unable to trace a total of nine studies, so we might have missed relevant study data. Furthermore, this systematic review was not supplemented by meta-analyses, which could have increased objectivity in interpreting results. However, that was not possible in the present study due to the small number of included studies (particularly per problem behaviour), and the heterogeneity of outcome measurements.

\section{Clinical message}

Amantadine may be useful in the treatment of irritability and aggression due to acquired brain injury

The safety of amantadine after brain injury appears to have been established.

Though this review did not include studies specifically concerned with determining side effects or adverse events, no major safety issues emerged from the included studies as long as there is an adequate creatinine clearance.

However, if used at all, a rigorous clinical evaluation should be performed, involving baseline assessment of target behaviour and, if possible, assessments after cessation of treatment. See Barlow et al. for possible designs (41).

\section{Conclusion}

This systematic review shows that amantadine may be efficacious on irritability and aggression after TBI. but not on executive function.

Amantadine is a safe drug in the presence of adequate creatinine clearance.

Future studies should use methodological designs that are suitable for the heterogeneous ABI population, like randomized Single Case Experimental Designs (SCEDs), and should include the effect of amantadine on societal participation and QoL.

\section{Declaration of interest}

The authors report no conflicts of interest

\section{ORCID}

C.M. Van Heugten (D) http://orcid.org/0000-0003-4272-7315

\section{References}

1. Williams WH, Evans JJ. Brain injury and emotion: an overview to a special issue on biopsychosocial approaches in neurorehabilitation. Neuropsychol Rehabil. 2003;13(1-2):1-11. doi:10.1016/S0960-9822(02)01374-X. Cited in PubMed: PMID: 22004099.

2. Eslinger PJ, Geder L. Behavioral and emotional changes after focal frontal lobe damage. In: Bogousslavsky J, Cummings JL, editors. Behavior and mood disorders in focal brain lesions. Cambridge: Cambridge University Press; 2000. p. 217-60.

3. Deb S, Lyons I, Koutzoukis C. 1999. Neurobehavioural symptoms one year after a head injury. Br J Psychiatry 174:360-65. Cited in PubMed: PMID:10533556.

4. Starkstein SE, Fedoroff JP, Price TR, Leiguarda R, Robinson RG. 1993. Apathy following cerebrovascular lesions. Stroke 24:1625-30. Cited in PubMed: PMID: 8236333.

5. Angelelli P, Paolucci S, Bivona U, Piccardi L, Ciurli P, Cantagallo A, Antonucci G, Fasotti L, Di Santantonio A, Grasso MG, et al. Development of neuropsychiatric symptoms in poststroke patients: a cross-sectional study. Acta Psychiatr Scand. 2004;110:55-63. doi:10.1111/j.1600.0447.2004.00297.x. Cited in PubMed: PMID: 15180780.

6. Tateno A, Jorge RE, Robinson RG. Clinical correlates of aggressive behavior after traumatic brain injury. J Neuropsychiatry Clin Neurosci. 2003;15:155-60. doi:10.1176/jnp.15.2.155. Cited in PubMed: PMID: 12724455.

7. Yudofsky SC, Silver JM, Jackson W, Endicott J, Williams D. The Overt Aggression scale for the objective rating of verbal and physical aggression. Am J Psychiatry. 1986;143:35-39. doi:10.1176/ajp.143.1.35. Cited in PubMed: PMID: 3942284.

8. Azouvi P, Jokic C, Attal N, denys P, Bussel B. 1999. Carbamazepine in agitation and aggressive behaviour following severe closed head injury: results of an open trial. Brain Inj 13:797-804. Cited in PubMed: PMID: 10576463.

9. Ashman TA, Gordon WA, Contor JB, Hibbard MR. Neurobehavioral consequences of brain injury. Mount Sinai J Med. 2006;73:999-1005. Cited in PubMed: PMID: 17195886.

10. Sander AM. Brain injury and the family. Neurorehabilitation. 2007;22:1-2.

11. Alves Gavao MG, Rocha Crispino Santos MA, Alves Da Cunha MJ. Amantadine and rimantadine for influenza A in children and the elderly. Cochrane Database Syst Rev. 2014 Nov 21;11:CD002745. doi: 10.1002/14651858.CD002745. Cited in PubMed: PMID: 25415374

12. Crosby N, Deane AH, Clarke CE. Amantadine in Parkinson's disease. Cochrane Database Syst Rev. 2003;1:CD003468. doi:10.1002/14651858.CDoo3468. Cited in PubMed: PMID: 12535476.

13. Arciniegas DB, Topkoff J, Silver JM. 2000. Neuropsychiatric aspects of traumatic brain injury. Curr Treatments Options Neurol 2:169-86. Cited in PubMed: PMID: 11096746.

14. Chew E, Zafonte RD. Pharmacological management of neurobehavioural disorders following traumatic brain injury-a state-ofthe-art review. J Rehabil Res Dev. 2009;46(6):851-79. Cited in PubMed: PMID: 12676415.

15. Deb S, Crownshaw T. The role of pharmacotherapy in the management of behaviour disorders in traumatic brain injury patients. 
Brain Inj. Cited in PubMed: PMID: 146602332004 Jan;18 (1):1-31. doi: 10.1080/0269905031000110463.

16. Fleminger S, Greenwood RJ, Oliver DL. Pharmacological management for agitation and aggression in people with acquired brain injury. Cochrane Database Syst Rev. 2006. Oct 18;(4):CD003299. doi:10.1002/14651858.CD003299.pub2. Cited in PubMed; PMID: 17054165.

17. Leone $\mathrm{H}$, Polsonetti BW. Amantadine fort traumatic brain injury: does it improve cognition and reduce agitation? J Clin Pharm Ther. Cited in PubMed: PMID: 158111612005 Apr;30(2):101-04. doi: 10.1111/j.1365-2710.2005.00628.x.

18. Levy M, Berson A, Cook T, Bollegala N, Seto E, Tursanski S, Kim J, Sockalingam S, Rajput A, Krishnadev N, et al. Treatment of agitation following traumatic brain injury: a review of the literature. Neurorehabil. 2005 Cited in PubMed: PMID: 16403996;20(4):279-306.

19. Warden DL, Warden DL, Gordon B, McAllister TW, Silver JM, Barth JT, Bruns J, Drake A, Gentry T, Jagoda A, et al. Guidelines for the pharmacologic treatment of neurobehavioral sequelae of traumatic brain injury. J Neurotrauma. Cited in PubMed: PMID: 170204832006 Oct;23(10):1468-501. doi: 10.1089/neu.2006.23.1468.

20. Lane-Brown A, Tate R. Interventions for apathy after traumatic brain injury (review). Cochrane Database Syst Rev. 2009;(2):CD006341. doi:10.1080/09602010902949207. Cited in PubMed: PMID: 19533496.

21. Karli DC, Burke DT, Kim HJ, CALVANIO R, FITZPATRICK M, TEMPLE D, MACNEIL M, PESEZ K, LEPAK P. Case study: effects of dopaminergic combination therapy for frontal lobe dysfunction in traumatic brain injury rehabilitation. Brain Inj. 1999 Cited in PubMed: 9972445;13:63-68. doi:10.1080/026990599121908.

22. Sami MB, Faruqui R. The effectiveness of dopamine agonists for treatment of neuropsychiatric symptoms post brain injury and stroke. Acta Neuropsychiatr. 2015 Apr 8;1-11. doi:10.1017/ neu.2015.17. Cited in PubMed: PMID: 25850757.

23. Hammond FM, Bickett AK, Norton JH, Pershad R. Effectiveness of amantadine hydrochloride in the reduction of chronic traumatic brain injury irritability and aggression. J Head Trauma Rehabil. 2014;29 (5):391-99. doi:10.1097/01.HTR.0000438116.56228.de. Cited in PubMed: PMID: 24263176.

24. Moher D, Liberati A, Tetzlaff J, Altman DG; The PRISMA Group. 2009. Preferred reporting items for systematic reviews and meta-analyses: the PRISMA statement. PLoS Med;6(7):e1000097. doi:10.1371/journal.pmed.1000097. Cited in PubMed: PMID: 19621072.

25. Spieth PM, Kubash AS, Penzlin AI, Illigens BM, Barlinn K, Siepmann T. Randomized controlled trials - a matter of design. Neuropsychiatr Dis Treat. 2016 jun 10;12:1341-49. doi: 10.2147/ NDT.S101938. Cited in PubMed: PMID: 27354804

26. Quality Assessment Tool for Quantitative Studies (QATFQS) from the Effective Public Health Practice Project. 2007 (EPHPP) (Thomas, Ciliska, Dobbins, \& Micucci, 2004, Deeks et al., 2003). doi:10.1094/PDIS-91-4-0467B

27. Tate RL, McDonald S, Perdices M, Togher L, Schultz R, Savage S. Rating the methodological quality of single-subject designs and n-of-1 trials: introducing the Single-Case Experimental Design (SCED) scale. Neuropsychol Rehabil. 2008;18(4):385-401. doi:10.1080/ 09602010802009201. Cited in PubMed: PMID: 18576270.

28. Hammond FM, Sherer M, Malec JF, Zafonte RD, Whitney M, Bell K, Dikman S, Bogner J, Mysiw J, Pershad R. Amantadine effect on perceptions of irritability after traumatic brain injury: results of the amantadine irritability multisite study. J Neurotrauma. 2015 Aug 15;32(16):1230-38. doi: 10.1089/ neu.2014.3803. Cited in PubMed: PMID: 25774566

29. Hammond FM, Malec JF, Zafonte RD, Sherer M, Bogner J, Dikmen S, Whitney MP, Bell KR, Perkins SM, Moser EA. Potential impact of amantadine on aggression in chronic brain injury. J Head Trauma Rehabil. Cited in PubMed: PMID:
288919082017 Sep/Oct;32(5):308-18. doi: 10.1097/ HTR.0000000000000342.

30. Hammond FM, Sherer M, Malec JF, Zafonte RD, Dikmen S, Bogner J, Bell KR, Barber J, Temkin N. Amantadine did not positively impact cognition in chronic traumatic brain injury: a multi-site, randomized controlled trial. J Neurotrauma. 2018 Oct 01;35(19):2298-305. doi: 10.1089/neu.2018.5767. Cited in PubMed: PMID: 29742960

31. Schneider WN, Drew-Cates WJ, Wong TM, Dombovy ML. Cognitive and behavioural efficacy of amantadine in acute traumatic brain injury: an initial double-blind placebo-controlled study, Cited in PubMed: PMID: 10579658. Brain Inj. Nov 1999;13(11):863-72.

32. Reekum RV, Bayley M, Garner S, Burke IM, Fawsett S, Hart A, Thompson W. 1995. N of 1 study: amantadine for the amotivational syndrome in a patient with traumatic brain injury. Brain Inj 9:49-53. Cited in PubMed: PMID: 7874096.

33. Kraus MF, Maki PM. Effect of amantadine hydrochloride on symptoms of frontal lobe dysfunction in brain injury: case studies and review. J Neuropsychiatry Clin Neurosci. Cited in PubMed: PMID: 9144101 1997;9(2):222-30. Spring. doi: 10.1176/jnp.9.2.222.

34. Arciniegas DB, Frey KL, Anderson CA, Brousseau KM, Harris SN. Amantadine for neurobehavioural deficits following delayed post-hypoxic encephalopathy, Cited in PubMed: OMID: 15666573. Brain Inj. Dec 2004;18(12):1309-18.

35. Kraus MF, Maki P. The combined use of amantadine and 1-dopa/ carbidopa in the treatment of chronic brain injury, Cited in PubMed: PMID: 9171930. Brain Inj. Jun 1997;11(6):455-60.

36. Kraus MF, Smith GS, Butters M, Donnell AJ, Dixon E, Yilong C, Marion D. Effects of the dopaminergic agent and NMDA receptor antagonist amantadine on cognitive function, cerebral glucose metabolism and D2 receptor availability in chronic traumatic brain injury: a study using positron emission tomography (PET), Cited in PubMed: PMID: 16134735. Brain Inj. Jul 2005;19(7):471-79.

37. Nickels JL, Schneider WN, Dombovy ML, Wong TM. Clinical use of amantadine in brain injury rehabilitation, Cited in PubMed: PMID: 7849690. Brain Inj. Nov-Dec 1994;8(8):709-18.

38. Tate RL, Perdices M, Rosenkoetter U, Wakim D, Godbee K, Togher L, McDonald S. Revision of a method quality rating scale for single-case experimental designs and n-of- 1 trials: the 15-item risk of bias in N-of-1 trials (RoBiNT) scale. Neuropsychol Rehabil. 2013;23(5):619-38. doi:10.1080/09602011.2013.824383. Cited in PubMed: PMID: 24050810.

39. Perdices M, Tate RL. Single-subject designs as a tool for evidence-based clinical practice: are they unrecognised and undervalued? Neuropsychol Rehabil. 2009;19(6):904-27. doi:10.1080/ 09602010903040691. Cited in PubMed: PMID:19657974.

40. Barlow DH, Nock MK, Hersen M. Single case experimental designs. Strategies for studying behavior change. Third ed. New York: Pearson Education, Inc; 2009.

41. Stunnenberg BC, Woertman W, Raaphorst J, Statland JM, Griggs RC, Timmermans J, Saris CG, Schouwenberg BJ, Groenewoud HM, Stegeman DF, et al. Combined N-of-1 trials to investigate mexiletine in non-dystrophic myotonia using a Bayesian approach; study rationale and protocol. BMC Neurol;2015. Open access. doi:10.1186/s12883-015-0294-4. Cited in PubMed: PMID: 25880166.

42. Zucker DR, Schmid CH, McIntosh MW, D'Agostino RB, Selker HP, Lau J. Combining single patient (N-of-1) trials to estimate population treament effects and to evaluate individual patient responses to treatment. J Clin Epidemiol. 1997;50 (4):401-10. Cited in PubMed: PMID: 9179098.

43. Zucker DR, Ruthazer R, Schmid CH, Feuer JM, Fischer PA, Kieval RI, Mogavero N, Rapoport RJ, Selker HP, Stotsky SA, et al. Lessons learned combining N-of- 1 trials to access fibromyalgie therapies. J Rheumatol. 2006;33:2069-77. Cited in PubMed: PMID: 17014022. 


\section{Appendix A.}

Searchstring pubmed 28-8-2018 146 hits

((( OR cerebrovascular disorders[mesh terms] OR brain neoplasms [mesh terms] OR hypoxia, brain [mesh terms] OR encephalitis [mesh terms] or hydrocephalus [mesh terms] Or meningitis [mesh terms] OR hypoxia [MeSH Terms] OR brain disease ${ }^{*}$ [Text Word] OR brain injur ${ }^{\star}[$ text word] OR TBI [text word] OR ABI [text word] OR brain damage [text word] OR head injur ${ }^{\star}$ [text word] or head-injur ${ }^{\star}$ [text word] OR stroke ${ }^{\star}$ [Text Word] OR CVA [text word] OR cerebrovascular accident ${ }^{\star}$ [text word] OR brain tumor [Text Word] OR brain tumors [text word] OR brain tumour [Text Word] OR brain tumours [Text Word] OR hypox ${ }^{*}$ [text word] OR encephalitis [Text Word] OR hydrocephal ${ }^{*}$ [Text Word] OR meningitis [text word] OR hypox* [text word])) AND (behavioral symptoms [MeSH Terms] OR apathy [Mesh Terms] OR anger [Mesh Terms] OR irritable mood [Mesh Terms] OR neurobehavioral manifestations [Mesh Terms] OR mental disorders [Mesh Terms] OR executive function [Mesh Terms] OR social participation [Mesh Terms] or Quality of life [Mesh Terms] or aggress* [text word] or apath* [text word] OR agitat* [text word] OR anger $^{*}$ [text word] OR angry [text word] OR irritab $^{*}$ [text word] OR behavio* [text word] OR executive function* [text word] OR dysexecutive syndrome [text word] OR participat ${ }^{\star}$ [text word] OR quality of life [text word] OR emotional lability [text word])) AND (amantadine [MeSH Terms] OR amantadine [text word])) NOT (neurodegenerative diseases [Mesh Terms] OR dementia [Mesh Terms])

searchstring embase 28-8-2018 398 hits

Line 1

Exp cerebrovascular disease/OR Exp brain injury/OR exp head injury/ OR exp brain tumor/OR Exp brain hypoxia/OR Exp encephalitis/OR exp hydrocephalus/OR exp meningitis/OR exp hypoxia/OR brain disease*. mp. OR brain injur*.mp. OR TBI .mp. OR ABI .mp. OR brain damage. $\mathrm{mp}$. OR head injur ${ }^{\star} \cdot \mathrm{mp}$. OR head-injur ${ }^{\star} \cdot \mathrm{mp}$. OR stroke*.mp. OR CVA . $\mathrm{mp}$. OR cerebrovascular accident ${ }^{\star}$.mp. OR brain tumor .mp. OR brain tumors .mp. OR brain tumour .mp. OR brain tumours .mp. OR encephalitis .mp. OR hydrocephal ${ }^{\star} . m p$. OR meningitis .mp. OR hypox ${ }^{\star} . m p$.

Line 2

Exp aggression/OR exp apathy/OR exp agitation/OR exp irritability/OR exp behavior disorder/OR exp problem behavior/OR exp executive function/OR exp social participation/OR exp Quality of life/OR exp mental instability/OR aggress ${ }^{\star}$.mp. or apath ${ }^{\star} . m p$. OR agitat ${ }^{\star} \cdot m p$. OR anger ${ }^{\star}$.mp. OR angry.mp. OR irritab*.mp. OR behavio ${ }^{\star}$.mp. OR executive function ${ }^{\star}$. $\mathrm{mp}$. OR dysexecutive syndrome.mp. OR participat ${ }^{\star} . \mathrm{mp}$. OR quality of life.mp. OR emotional lability.mp.

Line 3

Exp Amantadine/OR amantadine.mp.

Line 4

1 And 2 and 3

Line 5

limit 4 to (conference abstract or conference paper or "conference review")
Line 6

4 NOT 5

Line7

Exp dementia/or exp degenerative disease

Line 8

6 NOT 7

searchstring CINAHL 28-8-2018 61 hits

Line1

(MH "Brain Injuries+") OR (MH "Head Injuries+") OR (MH "cerebrovascular disorderst") OR (MH "brain neoplasmst") OR (MH "hypoxia, brain+") OR (MH "encephalitis+") OR (MH "hydrocephalus+") OR (MH "meningitist") OR (MH "anoxia+") OR brain disease* OR brain injur ${ }^{\star}$ OR TBI OR ABI OR head injur ${ }^{\star}$ or head-injur ${ }^{\star}$ OR stroke ${ }^{\star} \mathrm{OR}$ CVA OR cerebrovascular accident* OR brain tumor OR brain tumors OR brain tumour OR brain tumours OR hypox ${ }^{\star}$ OR hydrocephal ${ }^{\star}$ OR meningitis OR hypox*

Line 2

(MH "Behavioral symptomst") OR (MH “Apathy+") OR (MH "Neurobehavioral Manifestationst") OR (MH "behavioral and mental disorders+") OR (MH "executive function+") OR (MH "social participation+") OR (MH "quality of life+") OR aggress* OR apath* OR agitat* OR anger ${ }^{\star}$ OR angry OR irritab ${ }^{*}$ OR behavio* ${ }^{*}$ OR executive function ${ }^{*}$ OR dysexecutive syndrome OR participat ${ }^{\star}$ OR quality of life OR emotional lability

Line 3

(MH “amantadine+") OR amantadine

$\mathrm{N}=76$

Line 4

NOT

(MH "neurodegenerative diseases+") OR (MH “dementia+")

\section{Appendix B.}

Cumulative mention of side effects/adverse effects over the included studies:

gastrointestinal complains $(\mathrm{n}=19)$, change in appetite $(\mathrm{n}=9)$, sleep disturbance $(\mathrm{n}=9)$, hypoarousal/somnolence $(\mathrm{n}=4)$, hypomania $(n=2)$, tremors/shakes $(n=7)$, headache $(n=5)$, pain $(n=8)$, problems with orientation $(n=4)$, hallucinations $(n=3)$, executive function impairment $(n=1)$, other cognitive/behavioural/emotional $(n=9)$, sexual problems $(\mathrm{n}=3)$, infections $(\mathrm{n}=3)$, urine retention $(\mathrm{n}=1)$, light headedness/dizziness $(n=2)$, dry mouth $(n=2)$, dermatological $(n=3)$, seizures $(n=4)$, pedal edema $(n=1)$, other motor problems $(n=1)$, visual/ophthalmologic $(n=2)$, other $(n=15)$. 